\title{
Un dispositif innovant de conseil pédagogique : la visite de classe formative
}

\section{Alain Lerouge}

\section{(C) OpenEdition}

1 Journals

\section{Édition électronique}

URL : http://journals.openedition.org/trema/1389

DOI : 10.4000/trema.1389

ISSN : 2107-0997

\section{Éditeur}

Faculté d'Éducation de l'université de Montpellier

\section{Édition imprimée}

Date de publication : 1 janvier 2003

Pagination : 55-78

ISSN : 1167-315X

\section{Référence électronique}

Alain Lerouge, "Un dispositif innovant de conseil pédagogique : la visite de classe formative », Tréma [En ligne], 20-21 | 2003, mis en ligne le 06 octobre 2010, consulté le 30 avril 2019. URL : http:// journals.openedition.org/trema/1389; DOI : 10.4000/trema.1389

Ce document a été généré automatiquement le 30 avril 2019

Trema 


\title{
Un dispositif innovant de conseil pédagogique : la visite de classe formative
}

\author{
Alain Lerouge
}

\section{Introduction}

1 Le principe d'axer la formation des enseignants sur l'analyse de leurs pratiques spontanées conduit à repenser totalement l'articulation entre la demande de formation qui émerge du terrain et les formations didactiques et pédagogiques dispensées à l'IUFM (Lerbet-Sereni, F.; Violet D., 1999). Cela suppose de dépasser les scissions existantes entre généralistes, didacticiens et conseillers pédagogiques pour développer de véritables équipes intégrées, acceptant de travailler sur l'action située du stagiaire en formation.

2 L'enjeu est alors de développer la dimension professionnalisante de l'analyse de la pratique (Altet, 2000), dans le sens d'accompagner le transfert professionnel des contenus de la formation théorique. Cette perspective suppose que soient développés des dispositifs intégrateurs des différents volets de la formation répondant à une double fonctionnalité :

- d'une part, assurer l'émergence des logiques intrinsèques de l'action située ;

- d'autre part, permettre de modéliser ces logiques, en s'appuyant sur les contenus extrinsèques à l'action apportés par la formation théorique dans ses divers aspects.

Dans le cadre général de l'ethnométhodologie (Coulomb, 1993) qui insiste sur l'aspect émergent de l'organisation de la réalité sociale, et de l'ergonomie cognitive (Theureau, 1992), qui met l'accent sur la nécessité d'étudier des couplages " action situation », nous empruntons à Y. Chevallard (1997) la notion de praxéologie comme modèle intégrateur de cette double fonctionnalité.

Inscrit dans cette perspective, le dispositif « visite formative » a pour objectif essentiel de faire en sorte que les enseignants stagiaires intègrent au niveau de leur habitus 
professionnel, les contenus épars qu'on leur propose en formation. Tel que nous le décrivons, il est l'aboutissement d'un cycle d'expérimentations de quatre années scolaires portant sur deux groupes de formation sur lesquels nous avions toute liberté d'intervention tant auprès des stagiaires qu'en formation des formateurs. Le principe conducteur de cette recherche était l'ajustement successif, chaque année donnant lieu à une nouvelle phase expérimentale optimisant à la fois le dispositif de la visite et le système général de formation dans lequel il s'intègre. Après cette phase de recherche, les visites formatives ont été implantées dans diverses filières de l'IUFM, où elles sont généralement considérées par les formateurs et les stagiaires comme un bon vecteur d'accompagnement du transfert professionnel.

\section{Intégration du dispositif dans les filières de formation}

5 Le choix des groupes expérimentaux a été fait pour aborder l'ensemble de la problématique des formations premier et second degré dans leurs aspects didactiques et transversaux. Cela nous a amené à travailler l'intégration du dispositif «visite formative » dans deux groupes de vingt à vingt cinq stagiaires selon les années :

- un groupe de formation didactique second degré, pratiquement le groupe de formation des Professeurs de Lycée Collège de mathématiques (PLC2);

- un groupe de formation générale des Professeurs d'École (PE2).

6 Dans les deux cas, nous avons constitué des équipes intégrées de formateurs, en associant aux enseignants de l'IUFM les conseillers pédagogiques tuteurs pour le second degré, et les maîtres formateurs pour le premier degré. La contrainte des périodes de stagiarisation étant différente dans les deux filières, le dispositif a dû être intégré de manière spécifique à la planification de l'alternance dans chacune de ces filières.

\subsection{Intégration dans la formation des Professeurs de Lycée Collège}

7 Dans la filière des Professeurs de Lycée Collège, le rapport au terrain est fortement contraint par le stage en responsabilité organisé en continu dans une même classe pendant un an. Cette contrainte amène à organiser les visites formatives en lien avec le démarrage de ce stage, en échelonnant ces visites sur les deux premiers mois de l'année qui sont cruciaux pour la prise en main de la classe. Pratiquement, nous fonctionnons de la manière suivante sur un emploi du temps souple déterminé par les disponibilités des différents participants :

- chaque stagiaire du groupe de formation propose une date pour accueillir dans sa classe quatre à six de ses collègues, en veillant à ce que son conseiller pédagogique soit disponible à ce moment là ;

- les autres stagiaires s'inscrivent comme observateurs dans au moins quatre visites en fonction de leurs disponibilités propres ;

- trois formateurs IUFM se répartissent les visites ainsi arrêtées, et prennent en charge le suivi personnalisé des stagiaires qu'ils observeront au cours de ces visites.

L'intérêt majeur de cette organisation réside dans le fait que sur les premiers mois de l'année, chaque stagiaire assiste au moins à quatre séances de classe analysées comme nous allons le présenter, en étant à tour de rôle l'enseignant observé. Ces séances 
intègrent d'emblée le rapport au terrain à la formation théorique, et permettent dès le début de l'année scolaire de lancer de manière efficace la coordination avec les conseillers pédagogiques. Par ailleurs, le fait que les formateurs participants soient les tuteurs de personnalisation des stagiaires visités engage in situ le bilan de compétences professionnelles nécessaire au lancement de cette personnalisation.

Le tableau suivant synthétise cette organisation(Cf. tableau 1).

\begin{tabular}{|c|c|c|c|}
\hline \multicolumn{4}{|c|}{ Insertion dans la formation des Professeurs de Lycée et Collège } \\
\hline & 1er trimestre & $2^{\mathrm{e}}$ trimestre & $3^{\mathrm{e}}$ trimestre \\
\hline Stage & \multicolumn{3}{|c|}{ En continu sur l'ensemble de l'année } \\
\hline Cours & \multicolumn{3}{|c|}{ En continu sur l'ensemble de l'année } \\
\hline Visites & $\begin{array}{l}\text { Au moins quatre visites } \\
\text { formatives en groupes de } \\
\text { cinq stagiaires. }\end{array}$ & $\begin{array}{l}\text { Première visite } \\
\text { certificative }\end{array}$ & $\begin{array}{c}\text { Deuxième visite } \\
\text { certificative }\end{array}$ \\
\hline $\begin{array}{l}\text { Parcours } \\
\text { personnalisés }\end{array}$ & $\begin{array}{l}\text { Bilan de compétences } \\
\text { adossé aux visites formatives }\end{array}$ & \multicolumn{2}{|c|}{ Personnalisation de la formation } \\
\hline
\end{tabular}

Tableau 1

\subsection{Intégration dans la formation des Professeurs d'École} Directeur d'Ecole d'Application et de quatre Maîtres Formateurs. Chaque période de stage en responsabilité étant assortie de deux visites institutionnelles que nous n'avons pu modifier du fait de la complexité de leur gestion, nous avons pris le parti d'expérimenter les visites formatives sur les périodes de cours à l'IUFM, hors du temps de stagiarisation. le terrain, chacune de ces équipes réalisant une visite pendant la semaine qui suit la fin d'une période de stage. Cette organisation permet de travailler dans une classe connue du stagiaire accueillant, qui peut prévoir la séance observée de manière coordonnée avec le stage qu'il termine. Au total, chaque stagiaire participe sur l'ensemble de l'année à cinq visites formatives animées par un membre de l'équipe de formateurs de son groupe de formation générale. Pour l'une de ces visites, il est l'enseignant observé.

Le tableau suivant synthétise cette organisation (Cf. tableau 2).

\begin{tabular}{l|l|l|l|l|l|l|l|l|l|l|c}
\hline Insertion dans la formation des Professeurs d'École \\
\hline Périodes de cours & 1 & & 2 & & 3 & & 4 & & 5 & & 6 \\
\hline Périodes de stages & & 1 & & 2 & & 3 & & 4 & & 5 & \\
\hline Visites formatives & & & 1 & & 2 & & 3 & & 4 & & \\
\hline Visites certificatives & & & & $1-2$ & & & & & & $3-4$ & \\
\hline
\end{tabular}

Tableau 2 


\section{Le contrat de formation}

12 Si l'on souhaite utiliser la visite de classe comme creuset d'intégration de la formation dans des dispositifs d'alternance, il faut instituer cette démarche à partir d'un véritable contrat de formation. Deux aspects nous paraissent essentiels dans l'établissement de ce contrat : la finalisation réflexive de la démarche, et son positionnement par rapport à la certification professionnelle.

\subsection{Finalisation réflexive du dispositif}

13 En général, les stagiaires voient mal l'intérêt de l'analyse réflexive au regard de l'entretien conseil qu'ils jugent beaucoup plus efficace sur le court terme.

Leur demande de conseils se heurte à la démarche réflexive instituée par le formateur, et cette situation crée de l'incompréhension réciproque, qui se solde généralement par une maïeutique confuse dans laquelle le formateur cherche à faire émerger le « conseil » qu'il ne s'autorise pas à donner directement. Sans clarification du contrat de formation, l'analyse se développe sur un système d'attentes croisées qui lui retire toute son efficacité.

Pour éviter cette situation, il paraît essentiel de contractualiser le travail du groupe dans une perspective de construction de la réflexivité professionnelle. Cette contractualisation ne peut se faire qu'en affirmant clairement que le conseil et la construction de la capacité réflexive sont deux aspects de la formation qui ne peuvent être menées de pair sous peine de s'inhiber mutuellement. On peut alors justifier la finalisation réflexive de la visite formative par l'entraînement à la prise de décision dans la classe, en se référant à la nécessité de travailler sur la conscientisation de l'habitus professionnel (Perrenoud, 2001). Ce choix spécifique n'exclut pas le choix alternatif du conseil dans d'autres situations, ce qui rassure les stagiaires et les rend attentifs à la démarche.

\subsection{Sécurisation par rapport à la certification}

15 La visite de classe comme outil de formation est incompatible avec la nécessité de valorisation de soi induite par une perspective certificative. Il s'en suit la nécessité de contractualiser la formation et la certification sur deux types de visites faites par des formateurs différents. Cette séparation conduit à s'engager auprès des stagiaires sur le fait que le formateur assurant la visite certificative n'aura pas connaissance des observations formatives, et à repenser totalement la fonction du rapport de visite dans le cadre de la formation. C'est seulement au prix d'une confidentialité de ce rapport au regard de la certification que pourra s'établir la confiance indispensable à un travail sur les problèmes rencontrés.

\subsection{Positionnement du rapport de visite}

16 Sur ce principe de séparation des fonctions certificatives et formatives des visites de terrain, nous avons fait le choix de renoncer en visite formative au " rapport de visite » traditionnellement rédigé par le formateur, et de le remplacer par un exercice d'analyse 
de pratique par l'écriture réalisé par le stagiaire observé (Cifali M., 1995 et 1996). Nous proposons la rédaction d'un document en trois parties :

- un relevé de points qui ont pris sens au cours de l'analyse ;

- un projet personnel de formation contractualisé avec les formateurs ;

- et une nouvelle fiche de préparation de la leçon.

17 Cet exercice a essentiellement pour but de mettre en mots les repères professionnels abordés, et d'étayer le transfert de ces repères à d'autres contextes d'enseignement. Le texte produit sert de base au parcours personnalisé de formation, mais en aucune manière il n'est communiqué aux services administratifs qui gèrent la certification, ni aux formateurs qui en ont la charge.

\section{Le dispositif « visite formative »}

18 Le dispositif «visite formative " a pour objectif de proposer des "noyaux d'intégration théorie - pratique ", scandant la planification de la formation pour en étayer le transfert professionnel. Concrètement il met en œuvre une analyse intrinsèque de l'action d'un stagiaire sur le terrain de stage au cours d'une leçon observée par un groupes de pairs, et développe sur cette action une modélisation praxéologique adossée aux contenus théorique didactiques et généraux proposés à l'IUFM.

19 La séquence de travail est conçue dans une dynamique de formation réflexive du groupe des participants, tant pour celui qui fait la leçon que pour ceux qui l'observent. Elle dure environ une demi journée et se déroule sur trois phases :

- la préparation de l'observation;

- l'observation proprement dite ;

- et l'analyse de la pratique observée.

\subsection{Les participants}

20 L'expression "visite formative » désigne donc une séquence de formation construite autour d'une observation réalisée dans la classe d'un stagiaire, qui donne lieu à un travail collectif d'analyse de la pratique de ce stagiaire auquel participent six à huit personnes :

- le stagiaire accueillant qui fait la leçon;

- trois à six stagiaires observateurs ;

- le conseiller pédagogique du stagiaire accueillant ;

- et le formateur tuteur du parcours personnalisé du stagiaire accueillant chargé par ailleurs d'une partie de la formation théorique.

21 L'animation du dispositif est assurée soit par le conseiller pédagogique du stagiaire accueillant, soit par son tuteur de personnalisation. Il ne s'agit pas d'une co-animation, le rôle de chacun étant clairement défini : l'un anime, l'autre sert de personne ressource qui ne prend la parole que lorsqu'il y est invité par son collègue animateur. La participation conjointe du conseiller pédagogique et du formateur IUFM est très riche du point de vue de l'intégration de la visite aux versants théorique et pratique de la formation. Elle permet en particulier de mettre en place de manière coordonnée le projet de formation personnalisée du stagiaire visité. 


\subsection{Le type de séance observée}

Deux possibilités peuvent être envisagées pour la séance observée : elle peut avoir été préparée dans le cadre de la formation, ou au contraire être proposée spontanément par le stagiaire accueillant. Une séance préparée en formation présente l'avantage d'être bien dévolue aux observateurs, mais la préparation collective biaise la part personnelle de l'enseignant dans les phénomènes observés. A contrario, une leçon proposée par l'acteur est mal dévolue aux observateurs, mais permet d'analyser le cours d'action de l'enseignant en fonction de ses logiques propres. Dans la perspective de donner le primat à l'analyse de l'action située, nous avons privilégié cette seconde option.

\subsection{L'entretien qui prépare l'observation}

L'entretien qui prépare l'observation a pour fonction d'assurer la dévolution de la séance aux observateurs, et de répartir les tâches d'observation. Ce travail doit se faire sans déstabiliser l'enseignant qui fait la leçon, ce qui suppose de limiter les échanges à la seule prise d'informations en interdisant toute discussion critique sur les choix présentés.

\subsubsection{Dévolution de la séance aux observateurs}

La séance prévue par l'acteur (celui qui fait la leçon) est présentée au groupe des observateurs sur la base d'un tableau réalisé sur le modèle qui suit (cf. tableau 3).

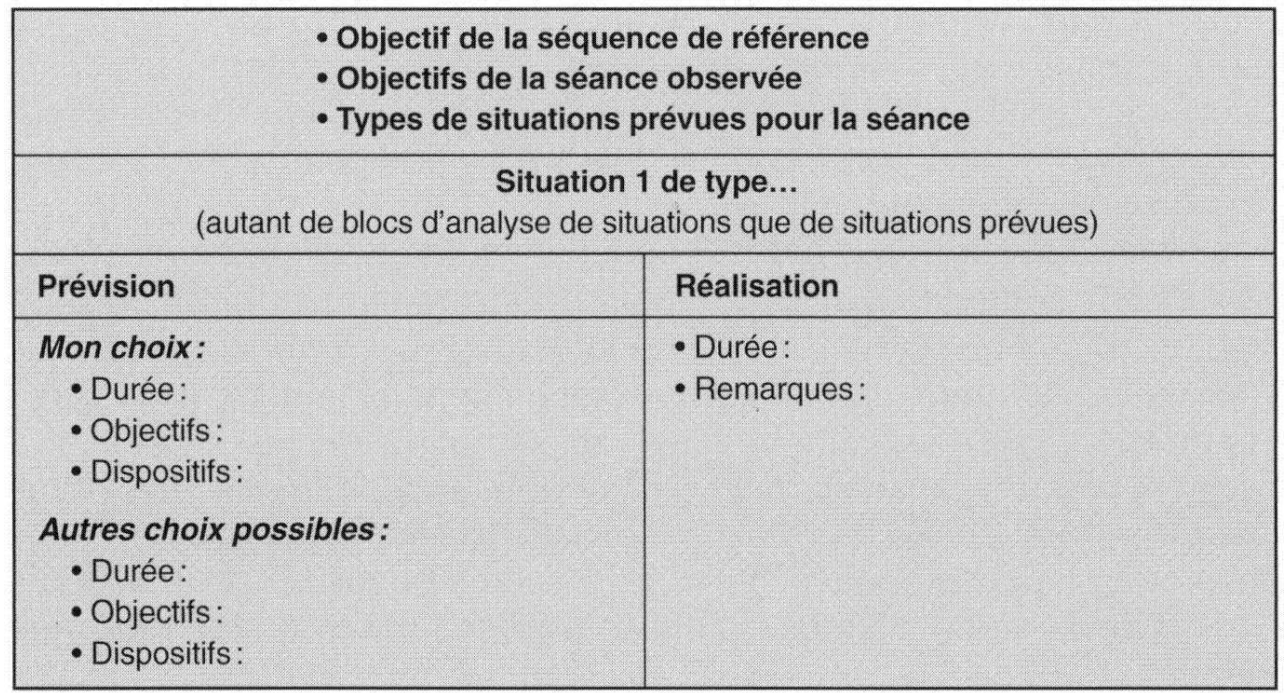

Tableau 3

Document d'analyse des situations prévues et réalisées.

Le bandeau initial et la partie gauche sont renseignés a priori par l'enseignant ; la partie droite est utilisée par les observateurs en cours d'observation pour rendre compte de la réalisation.

Ce tableau sert à la fois de support à l'entretien préparatoire (partie gauche), à l'observation (partie droite), puis dans son ensemble à l'analyse qui suit l'observation. Il est conçu dans la perspective de laisser à l'acteur le maximum de latitude tout en lui donnant une base de communication avec ses pairs. La seule contrainte qui lui est imposée est de présenter sa séance en termes de situations successives, une situation 
étant appréhendée comme une phase stabilisée du fonctionnement de la classe autour d'un objectif dominant et d'un dispositif approprié.

L'observation est ainsi focalisée sur des noyaux fonctionnels du point de vue de la logique prévisionnelle de l'acteur. Le bandeau initial situe globalement sa prévision dans une séquence de référence, et la disposition en deux colonnes qui suit permet de comparer localement cette prévision à la réalisation situation par situation. Plus particulièrement, la rubrique "autres choix possibles ", invite l'acteur à présenter sa réflexion sur des choix alternatifs, ce qui l'amène à relativiser ses options, et à engager les observateurs à en vérifier l'efficacité dans l'action. Au final, la séance est présentée par le biais d'une analyse contrastée des choix professionnels qui la sous-tendent, et non comme un objet figé dont le concepteur supporterait mal la critique.

\subsubsection{Répartition des tâches d'observation}

Les tâches d'observation sont réparties entre les stagiaires sur la base de trois entrées :

- le fonctionnement du dispositif prévu ;

- le jeu de l'habitus ${ }^{1}$ professionnel de l'enseignant ;

- l'activité des élèves.

Sur ces grands chapitres, chaque stagiaire spécifie ce qu'il envisage d'observer, et dont il rendra compte au groupe dans l'analyse qui suit la leçon. Parallèlement à l'observation du fonctionnement du dispositif prévu qui se fait sur la base du document que nous venons de présenter, le jeu de l'habitus de l'enseignant et l'activité des élèves donnent lieu à des protocoles d'observation spécifiques

L'observation du jeu de l'habitus de l'enseignant s'appuie sur une grille qu'il établit lui même en prévision de sa propre observation. L'auto-élaboration de cette grille engage sa réflexion sur les aspects problématiques de son comportement professionnel, et surtout l'amène à proposer des items d'observation sur lesquels il accepte a priori le jugement d'autrui. Pour guider la préparation de ce document, nous invitons le stagiaire à s'inspirer des items proposés par M. Postic dans l'ouvrage, Observation et formation des enseignants, 1977, p. 244-245, la consigne de ce travail étant de réduire le nombre de ces items à une dizaine, en les spécifiant sur des comportements professionnels qui manifestent l'habitus et qui dépassent le cadre local de la séance prévue.

Enfin l'observation des élèves est focalisée sur un ou deux élèves par observateur, désignés par l'enseignant à partir de critères cognitifs ou comportementaux. La tâche consiste à repérer les comportements d'intérêt et de désintérêt de ces élèves en proposant des hypothèses à caractère didactique ou pédagogique sur ces comportements. Ces hypothèses sont étayées par les productions écrites des élèves (cahiers de brouillon, de cours, d'exercices...) récupérées en fin de séance, et par un court entretien d'explicitation, réalisé lorsque c'est possible pendant environ un quart d'heure après la séance.

Le conseiller pédagogique et le formateur IUFM n'ont pas de tâche d'observation préalablement assignée, leur rôle consiste à repérer les noyaux autour desquels ils engageront l'entretien qui suit l'observation. 


\subsection{L'entretien qui suit l'observation}

33 L'objectif de cet entretien étant d'analyser l'action en situation, il est essentiel d'éviter toute modélisation prématurée qui aurait pour effet d'inhiber l'explicitation de cette action. Pour opérationnaliser le primat de l'analyse intrinsèque de l'action sur sa modélisation extrinsèque, nous proposons un protocole d'entretien en cycles « explicitation - modélisation », constitués de deux phases donnant lieu à des techniques d'animation spécifiques :

- une phase d'analyse intrinsèque de l'action visant à expliciter l'organisation en actes de l'enseignant (l'explicitation) ;

- suivie d'une phase d'analyse extrinsèque de cette organisation visant à développer un repérage théorique transférable (la modélisation). Ces phases sont clairement scindées dans le déroulement de l'entretien pour éviter leur inhibition réciproque.

\subsubsection{Animation des phases d'explicitation} du contexte de l'action située pour tirer de l'analyse intrinsèque qui vient d'être menée des repères professionnels transférables. La consigne est inversée: il ne s'agit plus d'entrer dans l'intrinsèque de l'action observée, mais de réagir par rapport à des «théories » personnelles que chacun applique à cette action pour se l'approprier. Cette seconde phase ouvre l'analyse sur le niveau théorique de la formation, l'enjeu étant de réaliser l'intégration de la culture professionnelle de chaque membre du groupe à la pratique observée et explicitée. C'est un moment clé de l'étayage du transfert professionnel qu'il faut éviter d'inhiber par un enfermement polémique avec l'acteur. Pour éviter cette dérive, la règle consiste à lui demander de ne pas intervenir pendant les phases de modélisation, en lui assurant un temps de réaction globale en fin de chacune de ces phases.

Pendant ces phases de modélisation, le rôle de l'animateur est crucial sur les effets de la formation. S'il reste trop en retrait, les débats du groupe risquent de ne pas sortir du contexte de la situation observée, et la modélisation nécessaire au transfert a du mal à se développer. Inversement, s'il intervient de manière trop cadrée, l'activité de modélisation est inhibée par la normalisation institutionnelle dont il est porteur aux yeux des stagiaires. Toute la pertinence de l'animation consiste à savoir se positionner dans ce paradoxe, en développant au sens vygotskien du terme, une zone proximale de formation 
dans laquelle les interventions du formateur prennent sens en appui de l'activité de modélisation des formés (Vygotski, 1934).

Ainsi, la question radicale de savoir si l'animateur doit rester en dehors des débats, ou au contraire apporter la dimension institutionnalisante de la formation est une fausse question. Il lui revient d'intervenir dans la proximité de ce qu'élabore le groupe, en conservant son statut de formateur. La position est difficile à tenir, d'autant que la pression du groupe va dans le sens de le contraindre à des réponses stéréotypées. S'il vise juste, et surtout s'il ne cherche pas à trop apporter de manière artificielle, il peut étayer très efficacement le processus de modélisation.

Une introduction possible de la phase de modélisation peut être de demander aux stagiaires de formuler les repères théoriques qu'ils jugent pertinents au regard de la situation analysée. Généralement, ces repères sont bousculés par la complexité de cette situation, et l'intervention de l'animateur dans le sens d'une clarification théorique de ces repères est alors tout à fait intéressante

\subsubsection{Animation du changement de phase}

39 Un autre aspect crucial du rôle de l'animateur concerne la manière dont il gère le changement de phase entre l'explicitation et la modélisation de l'action observée. Nous insistons sur le la nécessité de rompre l'équilibre installé dans la phase qui se termine, en évitant de laisser se développer un temps de pseudo continuité entre les deux phases : pas de phase intermédiaire, mais une discontinuité reconnue lorsqu'on change de paradigme de travail. Il est essentiel au changement de phase d'institutionnaliser clairement le nouveau contrat de formation, si ce n'est pas le cas, on observe généralement un croisement des attentes entre le formateur et les formés qui empêche la dévolution claire de ce nouveau contrat.

\subsubsection{Déroulement général de l'entretien} portant sur des aspects globaux ou parcellaires de la séance observée :

- il débute par deux ou trois cycles d'analyse locale portant sur des gestes ou des séquences de gestes significatifs ;

- et il se termine par un cycle d'analyse globale de la séance à la lumière des apports des différents cycles d'analyse locale.

En début d'entretien, l'animateur invite le groupe à ne pas chercher à couvrir de manière exhaustive l'ensemble de la prestation mais à se focaliser sur des séquences de gestes significatifs. Par la suite, en fonction des éléments apportés par le groupe, il est de son ressort de faire les choix de focalisation qu'il juge pertinents, et de lancer les cycles d'analyse autour de ces focalisations. Globalement sur l'ensemble de l'entretien, il ne doit pas se placer dans une relation confuse de parité, mais tenir la place qui lui est assignée pour chaque phase d'un cycle : accompagner l'explicitation de l'action par le jeu de miroir acteur - observateurs puis étayer en alternance la modélisation de repères professionnels.

Enfin, l'animateur a pour tâche d'accompagner le travail d'écriture demandé au stagiaire et la formation personnalisée qui en découle.

43

Nous ne développerons pas cet aspect qui est incident par rapport à l'animation du dispositif, mais qui le positionne dans la planification globale de la formation. 
Pour terminer la présentation de ce travail de recherche, nous allons maintenant développer ses fondements théoriques, tant au niveau de l'explicitation intrinsèque de l'action observée, qu'au niveau de la modélisation extrinsèque de cette action.

\section{4. Éléments sur l'explicitation de l'action}

L'analyse de pratique qui suit la leçon est structurée autour de cycles «explicitation modélisation" fondés sur la notion de praxéologie qu'Y. Chevallard (1997) définit au regard de quatre entrées :

- la tâche que s'assigne l'enseignant (son intention générale);

- les techniques qu'il développe (l'action située observable);

- les technologies qu'il fournit dans l'entretien (l'explicitation qu'il donne de son action);

- les théories de référence (le champ de la culture professionnelle extrinsèque à l'action).

Un cycle explicitation - modélisation vise à intégrer praxis et logos, en appréhendant le versant de l'explicitation par le jeu des trois premières entrées de la notion de praxéologie (tâche, technique, technologie), et le versant modélisation par la quatrième (théorie) positionné du côté de l'explicatif culturel, extrinsèque à l'action du sujet. L'ensemble est à appréhender de manière intégrée, ce que schématise le tableau qui suit en positionnant la notion de praxéologie à l'interface de l'action du sujet et de la culture professionnelle ${ }^{2}$ (Cf. tableau 4).

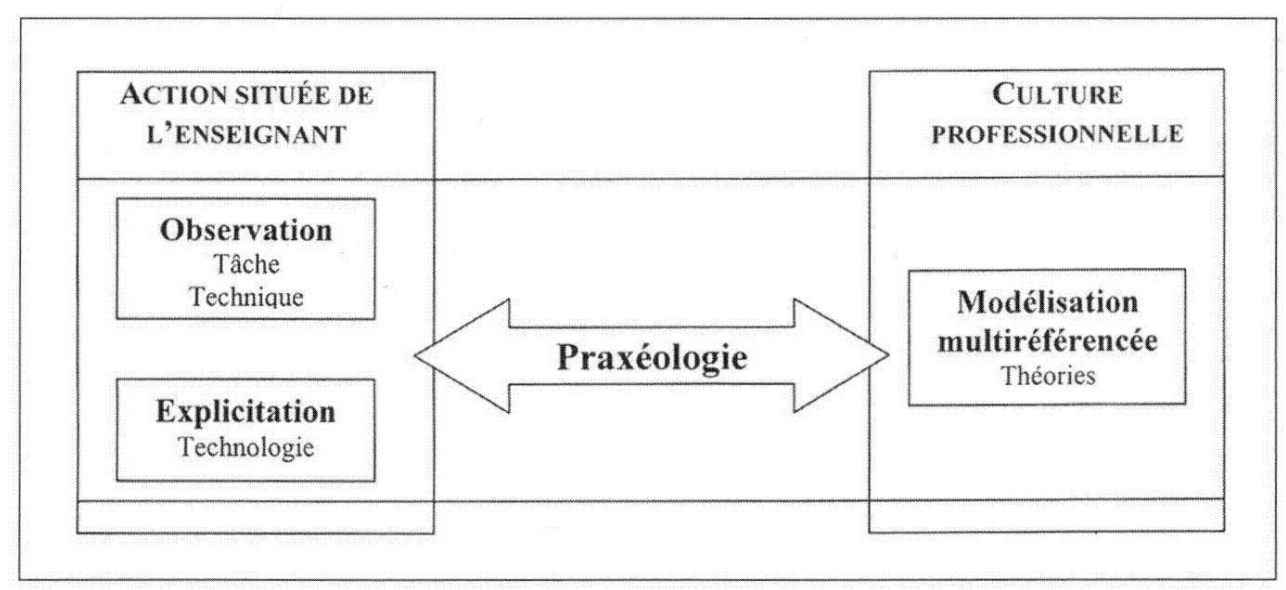

\section{TABLEAU 4}

La notion de praxéologie.

Dans ce cadre théorique général, deux niveaux d'organisation praxéologique paraissent particulièrement pertinents dans l'analyse du cours d'action d'un enseignant :

- le niveau des micro praxéologies de l'acteur (les gestes d'enseignement) : par exemple, le fait d'annoncer publiquement les notes en rendant les copies aux élèves ;

- et le niveau de macro praxéologies organisant une séquence de gestes au regard d'une fonction didactique ou pédagogique particulière : par exemple, au niveau didactique la macro praxéologie « situation problème ${ }^{3}$ ", ou au niveau pédagogique, la macro praxéologie « conseil des élèves ${ }^{4} »$. 


\subsection{La notion de "geste d'enseignement »}

47 Un «geste d'enseignement " est donc une micro praxéologie du cours d'action de l'enseignant, identifiée par une technique observable, et explicitée a posteriori par une technologie particulière. Il peut avoir un caractère coutumier lorsqu'il est fortement implanté dans l'habitus de l'acteur, ou avoir au contraire un caractère exceptionnel, notamment lorsqu'il manifeste une tentative de changement. Dans les deux cas, il apparait comme une unité élémentaire du cours de l'action située, qui peut être en accord ou en rupture avec le champ théorique extrinsèque apporté par la formation. Pour des raisons de commodités de communication avec les stagiaires, nous distinguons les gestes pédagogiques des gestes didactiques, par exemple :

- le fait d'écrire une définition au tableau est un geste d'enseignement à caractère didactique ;

- le fait d'élever la voix pour faire taire un élève est un geste d'enseignement à caractère pédagogique.

Cependant, il s'agit d'une distinction formelle. En effet, écrire une définition au tableau peut relever d'avantage de l'intention pédagogique de calmer les élèves en les contraignant à écrire, que de l'intention didactique de passer à une phase de synthèse. Ce geste peut d'ailleurs relever des deux intentions à la fois. La distinction entre didactique et pédagogique résulte de l'analyse intrinsèque de l'action et non d'un a priori extrinsèque sur cette action.

A titre d'exemple, nous analysons dans le tableau qui suit le geste d'enseignement "annoncer publiquement les notes en rendant les copies aux élèves" extrait d'un protocole d'entretien avec un stagiaire en formation (Cf. tableau 5). Dans ce cas, la théorie (culturel extrinsèque) est en rupture avec la technologie (personnel intrinsèque) avancée par l'acteur.

\begin{tabular}{|c|c|}
\hline \multicolumn{2}{|l|}{ Analyse d'un geste d'enseignement } \\
\hline \multicolumn{2}{|c|}{ Geste d'enseignement: « annoncer les notes en rendant les copies » } \\
\hline Analyse intrinsèque de l'action & Analyse extrinsèque de l'action \\
\hline $\begin{array}{l}\text { Tâche: Permettre aux élèves de se situer les uns par } \\
\text { rapport aux autres }\end{array}$ & $\begin{array}{l}\text { Théorie : Effet de la représentation de soi sur la réus- } \\
\text { site scolaire. }\end{array}$ \\
\hline $\begin{array}{l}\text { Technique: Donner la note à haute voix en rendant } \\
\text { la copie à l'élève. }\end{array}$ & $\begin{array}{l}\text { Remarque: Pour les élèves faibles, le champ théo- } \\
\text { rique est ici en rupture avec la technologie avancée }\end{array}$ \\
\hline $\begin{array}{l}\text { Technologie: La transparence des notes au niveau } \\
\text { de l'ensemble de la classe est une garantie d'équité } \\
\text { et de justice et permet une juste estimation de soi par } \\
\text { rapport aux autres. }\end{array}$ & par l'acteur. \\
\hline
\end{tabular}

TABLEAU 5

\subsection{La notion de " séquence d'enseignement »}

50 À un second niveau, nous prenons comme objet d'analyse de l'action de l'enseignant des " séquences d'enseignement ", c'est-à-dire des ensembles de gestes d'enseignement qui remplissent pour l'acteur une fonction dominante didactique ou pédagogique particulière (découverte d'une notion, maintien de l'autorité, etc.). Chronologiquement, une séquence d'enseignement peut être constituée :

- par une succession de gestes temporellement groupés : « séquence compacte »; 
- ou au contraire par une succession de gestes temporellement séparés : « séquence sérielle ».

51 Une séquence d'enseignement est donc une organisation praxéologique, identifiée par la dominante fonctionnelle que lui attribue l'acteur, et qui apparaît aux observateurs comme une succession de gestes coutumiers et de gestes exceptionnels. L'analyse de la distribution des gestes de la séquence, ouvre sur l'articulation de l'habitus et des effets de la formation.

\subsubsection{Repérage d'une séquence par les ruptures de contrat} l'enseignant, nous utilisons l'explicitation des ruptures de contrat didactique (G. Brousseau, 1989) ou de contrat pédagogique, en interprétant ces ruptures comme des indicateurs de modification fonctionnelle du processus d'enseignement. Ces ruptures peuvent apparaître sous forme technique (changement de comportement de l'acteur) ou sous forme technologique (changement de discours dans l'explicitation).

Localisée ainsi par les ruptures de contrat, une séquence d'enseignement est alors identifiable comme un ensemble de gestes d'enseignement, fédérés par le même contrat dans une finalité didactique ou pédagogique particulière. Il est à remarquer que le même contrat peut être repris sur des périodes séparées dans le temps, et que les séquences ainsi obtenues peuvent avoir un caractère compact ou sériel au sens ou nous venons de le définir.

Une telle séquence peut alors être analysée de manière globale, ou de manière chronologique en faisant apparaître l'enchâssement des gestes d'enseignement qui la constituent.

\subsubsection{Analyse globale et analyse chronologique du cours d'action de l'enseignant}

Deux sortes d'analyse de séquence peuvent être menées :

- une analyse globale qui prend pour objet la globalité de la séquence pour en expliciter les quatre entrées praxéologiques (tâche, technique, technologie, théorie) ;

- ou une analyse chronologique à partir d'un support vidéo, difficile à réaliser dans le temps de l'entretien, mais qui peut donner lieu à un travail ultérieur du stagiaire.

À titre d'exemple, le tableau suivant (Cf. tableau 6), présente l'analyse praxéologique globale d'une séquence d'enseignement visant à corriger une erreur rédhibitoire. Il est à remarquer que la théorie culturelle se scinde en deux champs dont l'un valide et l'autre invalide la technologie de l'acteur.

\begin{tabular}{|c|c|}
\hline \multicolumn{2}{|c|}{ Analyse globale d'une séquence d'enseignement } \\
\hline \multicolumn{2}{|c|}{ Séquencee d'enseignement: "corriger une erreur redhibitoire» } \\
\hline Analyse intrinsèque de l'action & Analyse extrinsèque de l'action \\
\hline $\begin{array}{l}\text { Tâche: Permettre aux élèves de ne plus faire une } \\
\text { erreur redhibitoire. }\end{array}$ & $\begin{array}{l}\text { Théorie : Conception béhavioriste et conception } \\
\text { constructiviste de l'apprentissage. }\end{array}$ \\
\hline $\begin{array}{l}\text { Technique : Faire énoncer la règle de référence par } \\
\text { un élève; donner une série d'exercices de manière. } \\
\text { magistrale. }\end{array}$ & $\begin{array}{l}\text { Remarque : La théorie béhavioriste valide la technolo } \\
\text { gie de l'acteur, alors que la théorie constructiviste } \\
\text { l'invalide. }\end{array}$ \\
\hline $\begin{array}{l}\text { Technologie: C'est la répétition qui crée la compré- } \\
\text { hension; la correction magistrale évite limpregnation } \\
\text { par des erreurs au tableau. }\end{array}$ & \\
\hline
\end{tabular}

TABLEAU 6 
En complément de l'analyse développée lors de la visite, et lorsqu'on dispose d'un film de la séance observée, on peut demander au stagiaire de reprendre dans son travail d'écriture l'analyse de cette séance au niveau de la chronologie des gestes qui la constituent. Ce travail peut se traiter sous forme d'un tableau du type suivant (Cf. tableau 7), faisant apparaître l'enchâssement des gestes isolés et des séquences compactes ou sérielles.

\begin{tabular}{|l|l|l|l|l|l|l|l|l|l|l|l|l|}
\hline Analyse chronologique de séquences d'enseignement \\
\hline Gestes d'enseignement & 1 & 2 & 3 & 4 & 5 & 6 & 7 & 8 & 9 & 10 & 11 & 12 \\
\hline Séquence compacte & & & & & & & & & & & & \\
\hline Séquence sérielle & & & & & & & & & & & & \\
\hline Gestes isolés & & & & & & & & & & & & \\
\hline
\end{tabular}

TABLEAU 7

58 Un entretien ultérieur ${ }^{5}$ avec le stagiaire autour de ce tableau s'avère alors souvent des plus intéressant.

\section{5. Éléments sur la modélisation de l'action}

Sur le versant de la modélisation de l'action, nous avons fait le choix de travailler dans un environnement théorique multi-référencé (J. Ardoino, 1993), le principe étant celui d'une approche co-disciplinaire (Cl. Blanchard Laville et coll., 1997), chaque champ théorique donnant un regard spécifique sur la pratique, sans chercher à intégrer ces divers champs dans une matrice explicative unique.

L'analyse intrinsèque du cours d'action étant faite en termes de fonctions didactiques ou pédagogiques, la cohérence amène à ouvrir l'analyse intrinsèque sur le versant théorique de ces fonctions. À une approche fonctionnelle intrinsèque correspond une approche fonctionnelle extrinsèque permettant $d$ 'analyser le caractère rarement univoque des gestes et des séquences d'enseignement. La démarche est la même, mais le référentiel change: ce n'est plus l'action du sujet qui sert de référence, mais la culture professionnelle extrinsèque à cette action.

61 Pour illustrer notre propos, nous présentons le repérage fonctionnel multi-référencé arrêté en formation d'enseignants de mathématique du second degré. Pratiquement, chacune des fonctions référencées dans le tableau qui suit (Cf. tableau 8), est abordée en formation théorique avec les stagiaires, et en formation de formateurs avec les conseillers pédagogiques. Ce travail préalable ouvre une base commune de communication qui permet le travail de modélisation lors des visites de classe. 


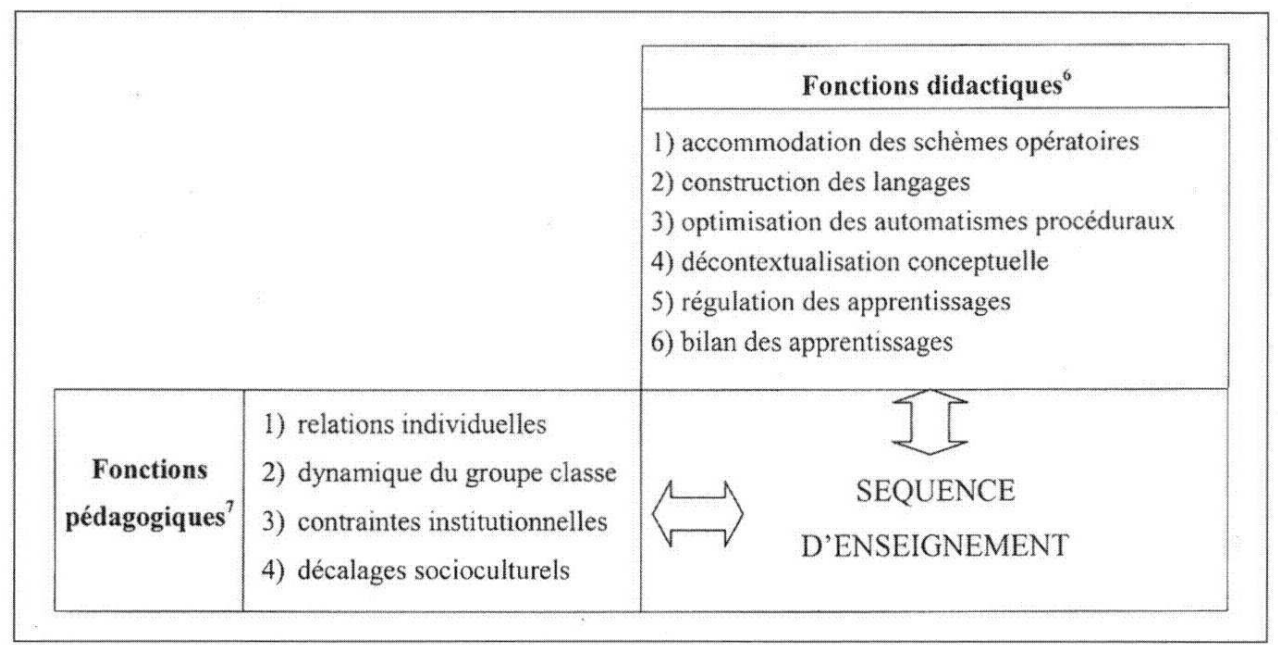

Repérage théorique multiréférencé d'une séquence d'enseignement. ${ }^{6}$ et ${ }^{7}$

TABLEAU 8

De manière pragmatique, nous avons cherché à stabiliser un repérage extrinsèque de l'action de l'enseignant qui soit adapté :

- aux connaissances théoriques des stagiaires en formation;

- à la culture professionnelle des conseillers pédagogiques qui les encadrent ;

- et à la prise compte de la complexité de l'action de l'enseignant, mêlant en permanence le didactique et le pédagogique.

Nous donnons dans les paragraphes qui suivent un développement point par point des diverses fonctions synthétisées dans le tableau ci-contre.

\subsection{Fonctions didactiques d'une séquence d'enseignement}

En référence aux travaux de didactique des mathématiques, et tout particulièrement à ceux de G. Brousseau $(1986,1989)$, Y. Chevallard $(1992,1997)$, R. Douady (1992) et G. Vergnaud $(1991,1994)$, nous proposons aux stagiaires de mathématiques d'analyser leur enseignement au regard de six fonctions didactiques théoriques :

- l'accommodation des schèmes opératoires : « mise en actes »;

- la construction des langages : « mise en mots»;

- l'optimisation des automatismes procéduraux : « mise en application »;

- la décontextualisation conceptuelle : « réinvestissement »;

- la régulation des apprentissages : « évaluation - régulation»;

- le bilan des apprentissages : « évaluation-bilan ».

\subsubsection{Fonction d'accommodation des schèmes opératoires : « mise en acte »}

En mathématiques, la fonction d'accommodation des schèmes opératoires a pour objet de faire apparaître des connaissances "en actes", c'est-à-dire des connaissances non verbalisables a priori (notion de théorème en actes au sens de G. Vergnaud, 1992). Il s'agit pour l'essentiel de mettre en réseau dans les séquences de démarrage d'un cycle d'apprentissage le « déjà là » en acte avec l'apprentissage visé.

La notion clé travaillée en formation est l'accommodation piagétienne, en insistant sur le fait qu'un exercice visant l'accommodation du système cognitif n'est pas une simple 
activité de découverte, mais un problème qui a pour fonction de montrer les insuffisances des connaissances installées, et d'en permettre la restructuration en acte. C'est une notion qui est désignée dans la littérature scolaire en termes de "situation problème ", mais l'expression « mise en acte » nous paraît plus adaptée, en ce sens qu'elle désigne des activités d'accommodation opératoire qui ne mobilisent pas de manière explicite les capacités langagières de l'élève.

\subsubsection{Fonction de construction des langages : « mise en mots »}

67 Si la «mise en acte» permet de créer du réseau cognitif, elle ne suffit pas à la conceptualisation des nouvelles connaissances qui restent contextualisées au cadre de leur production. Dans un second temps, l'objectif pour l'enseignant de mathématique est de faire aboutir le processus de conceptualisation en greffant sur une acquisition en actes les mots qui permettent de la signifier. La fonction de construction des langages vise à assurer cette étape indispensable en repérant un double niveau (G. Brousseau, 1986) :

- celui de la connotation en langage élève qui permet de valider les conceptions en actes (la formulation);

- et celui de la dénotation institutionnelle qui vise à construire le sens des expressions institutionnelles stabilisées par l'usage (l'institutionnalisation).

Généralement, l'étape de construction des langages est occultée par les enseignants de mathématiques, qui passent d'activités de découverte au psittacisme de l'apprentissage de règles supposées construites par ces activités. Il s'agit de les convaincre de la nécessité de construire sur les signifiés en actes, les signifiants conceptuels qui expriment les concepts émergeants.

69 Nous insistons sur l'importance de travailler la formulation avant l'institutionnalisation parce que cette démarche permet de construire le langage scientifique au regard du langage familier des élèves. Globalement, la fonction de construction des langages est souvent identifiée comme "fonction de synthèse ", mais l'expression «mise en mots" nous paraît mieux convenir parce qu'elle désigne une véritable activité de l'élève sur le langage, et non une simple mise en forme faite par l'enseignant.

\subsubsection{Fonction d'optimisation des automatismes procéduraux : « mise en application »}

70 Les concepts étant construits, à la fois au niveau des invariants opératoires et des signifiants langagiers (Vergnaud, 1991), il reste à optimiser les procédures spontanées des élèves pour installer des automatismes procéduraux efficients. Nous désignons cette fonction d'optimisation des automatismes procéduraux par l'expression «mise en application ». Il s'agit d'une activité tout à fait différente de la mise en acte, en ce sens qu'elle ne vise pas l'accommodation des schèmes cognitifs, mais leur stabilisation dans leur application à une classe de problèmes.

71 Le processus piagétien de référence est ici l'assimilation, la clé étant de distinguer les exercices qui permettent de remplir cette fonction de ceux qui visent l'accommodation des connaissances dans la phase de mise en acte. Si l'automatisation des schèmes opératoires est à l'évidence indispensable à la pérennisation des apprentissages, elle 
court-circuite souvent la conceptualisation par de la simple répétition. Nous insistons sur les aspects suivants :

- ne pas mettre un concept « en application » avant de l'avoir mis « en actes » et « en mots", sous peine d'inhiber la conceptualisation par de la répétition stérile;

- se garder d'un « bachotage » non finalisé, en fondant ce type de travail sur une analyse de l'erreur avec exercices de traitement différenciés.

\subsubsection{Fonction de décontextualisation conceptuelle : « réinvestissement »}

La fonction de réinvestissement consiste à faire en sorte que les élèves sache mobiliser un concept hors du champ de référence qui a permis de le construire. Pratiquement, il s'agit de leur apprendre à réinvestir un savoir acquis sur des questions qui n'évoquent pas spontanément ce savoir, ce qui permet de faire aboutir le processus de conceptualisation en désétayant le concept construit de son contexte de construction.

Généralement, cette fonction est mal assumée par les enseignants, qui jouent la rentabilité à court terme en développant fortement la mise en application (même contexte) au détriment du réinvestissement (changement de contexte). Il s'agit de les convaincre de faire travailler leurs élèves sur des "problèmes ouverts ", c'est-à-dire des problèmes dont l'énoncé ne mobilise pas le concept par analogie, mais oblige à l'activité de transfert.

\subsubsection{Fonctions de régulation des apprentissages}

74 En ce qui concerne l'évaluation, nous pensons important de repérer deux fonctions essentielles: la fonction de régulation des apprentissages (évaluation formative) et la fonction de bilan (évaluation sommative). Au niveau de la fonction de régulation des apprentissages, nous distinguons :

- la régulation par l'élève de ses propres apprentissages dans laquelle l'évaluation est utilisée comme outil de formation (évaluation formatrice);

- la régulation par l'enseignant des effets de son enseignement, dans laquelle l'évaluation est utilisée comme diagnostic externe à l'élève (évaluation diagnostique).

Le propre de l'évaluation formatrice est de «former» par l'évaluation, et donc de développer de l'apprentissage, alors que celui de l'évaluation diagnostique est simplement de prendre de l'information pour réguler la formation de l'extérieur. En d'autres termes, l'évaluation formatrice est une régulation qui boucle sur l'élève, l'évaluation diagnostique une régulation qui boucle sur l'enseignant.

\subsubsection{Fonction de bilan des apprentissages}

Parallèlement, nous distinguons trois aspects de la fonction de bilan selon qu'il s'agisse :

- d'un bilan au regard des progrès personnel de l'élève (évaluation différentielle) ;

- d'un bilan au regard d'un groupe de référence qui sert de norme relative (évaluation normative) ;

- ou enfin un bilan au regard d'un niveau de compétence socialement repéré qui sert de norme absolue (évaluation certificative).

Sur l'ensemble, nous insistons sur la nécessité de critérier l'évaluation, et d'optimiser les tests proposés aux critères sélectionnés. Enfin nous développons une réflexion docimologique en posant le principe que «la note » est un vecteur de communication 
sociale de la performance, et qu'il convient de s'interroger sur sa fonction lorsqu'elle est utilisée dans une autre finalité.

\subsection{Fonctions pédagogiques d'une séquence d'enseignement}

78 Les gestes d'enseignement sont des gestes complexes, rarement monoréférencés, qui sont le plus souvent produits par une prise de décision qui optimise divers paramètres du processus d'enseignement. Sur un geste précis les fonctions s'entremêlent, et la dimension didactique de l'action de l'enseignant est difficilement séparable de sa dimension pédagogique. Ce constat amène à croiser les six fonctions didactiques que nous venons de décrire à quatre fonctions pédagogiques respectivement focalisées sur :

- la gestion des relations individuelles ;

- la gestion de la dynamique du groupe classe ;

- la gestion des contraintes institutionnelles ;

- et enfin la gestion des décalages socio-culturels.

\subsubsection{Fonction de gestion des relations individuelles}

Les métiers d'éducation travaillent sur l'humanité des acteurs qui les assument, et à l'école comme partout ailleurs, les phénomènes de transfert sont la trame des relations interpersonnelles. La relation pédagogique se joue sur fond de résonances multiples entre l'histoire de l'enseignant et celle de l'élève, cette situation étant particulièrement sensible chez les jeunes enseignants qui ont généralement du mal à se mettre à distance de leur expérience d'élève (A. Lerouge, 1998). De manière pragmatique, pour étayer la gestion de ces phénomènes, nous travaillons le positionnement professionnel en insistant sur les points suivants :

- ne pas réagir en miroir ;

- nommer les élèves ;

- les respecter si l'on veut se faire respecter ;

- donner un projet de réussite ;

- n'exiger que ce que l'on peut tenir ;

- poser avec l'élève le sens d'une sanction, et donc son caractère nécessairement individuel ;

- rompre toute situation d'enfermement dans une mécanique de surenchère punitive qui se joue sur fond d'identité personnelle bafouée, en général sans issue pour les deux protagonistes ;

- et enfin « parler vrai » en toutes circonstances, ce qui ne signifie pas que l'on peut tout dire dans la classe, mais que si l'on ne veut pas répondre à une question, on le dit clairement plutôt que de raconter n'importe quoi.

Très concrètement, lors de situations de crise en relation duelle, nous invitons les enseignants à ne pas chercher à régler le problème dans l'arène de la classe, mais à provoquer un entretien différé avec l'élève, éventuellement médiatisé par un autre élève ou un autre adulte de l'établissement. Cela conduit à travailler en formation les entretiens de résolution de crise sous forme de jeux de rôle, dans l'objectif de sortir de la surenchère punitive pour contractualiser la relation pédagogique. Tout particulièrement, clarifier le fait que la relation parent - enfant n'est pas du même ordre que celle d'un enseignant avec ses élèves permet d'installer une relation pédagogique hors de la répétition stérile des scénarios familiaux. 


\subsubsection{Fonction de gestion de la dynamique du groupe classe}

81 Si pour une part la relation pédagogique s'installe au niveau des relations interpersonnelles, l'enseignant ne peut ignorer que dans son ensemble la dynamique du groupe classe relève de phénomènes psychosociaux non réductibles à la simple juxtaposition de ces relations. La professionnalité dans ce domaine consiste à savoir travailler la résistance au changement dans les groupes restreints, au niveau de la dynamique globale de ces groupes et non par traitement isolé des individus.

82 Ainsi, en situation de crise, contrairement à la gestion des relations individuelles traitées plutôt par entretiens différés, le traitement de la globalité de la dynamique du groupe classe consiste à travailler les représentations sociales du groupe par des situations de mise en débat collectif, en instituant des temps de parole inspirés des pratiques de la pédagogie institutionnelle (F. Imbert, 1994). Cet aspect de la relation pédagogique conduit à travailler sur l'appropriation par les élèves d'un règlement de fonctionnement de la classe et sur la mise en œuvre de situations de régulation lorsque ce règlement est bafoué.

\subsubsection{Fonction de gestion des contraintes institutionnelles}

83 Au-delà des phénomènes relationnels et des phénomènes de groupe, une situation de classe peut être éclairée par le système de contraintes institutionnelles dans lequel se trouvent l'enseignant et les élèves. Trois niveaux de contraintes apparaissent fréquemment :

- le niveau organisationnel de l'établissement (séance mal placée dans l'emploi du temps);

- le niveau de la contrainte des programmes (suivre le programme dans une classe faible);

- et enfin celui du statut institutionnel de l'enseignant, à la fois juge et partie dans le traitement des conflits dans la classe.

\section{Le rapport aux programmes}

La contrainte des programmes est récurrente dans les analyses de pratiques professionnelles, et l'obligation de suivi du programme institutionnel fait obstacle à la prise en compte par l'enseignant du niveau réel des élèves qui lui sont confiés. Cet aspect génère l'inadaptation des savoirs enseignés à la réalité de la classe et produit une situation structurelle de crise pédagogique impossible à traiter dans le seul contexte conjoncturel. Le dépassement de cet obstacle oblige l'enseignant à faire le deuil d'un même niveau de programme pour tous, à accepter le principe d'une évaluation différenciée, et à travailler en projet d'établissement pour donner à sa démarche une cohérence institutionnelle sur plusieurs années. Autant de points qui supposent une véritable révolution des mentalités installées.

\section{L'enseignant juge et partie}

85 Au niveau institutionnel global, l'absence de lieu de médiation dans le système éducatif, apparaît comme un véritable vide institutionnel dans la gestion des situations de crise. En particulier, le statut de l'enseignant à la fois juge et partie dans ses différents avec les élèves, développe une violence institutionnelle qui diffuse dans la relation pédagogique (B. De France, 1995). Dans ce contexte, enseignants et élèves se vivent comme victimes de contraintes institutionnelles qu'ils subissent avec la conviction de ne pouvoir rien y 
changer. Il s'agit alors de traiter en formation cette représentation passive des acteurs dans le système éducatif, en travaillant sur le pouvoir instituant des enseignants au sein de projets d'établissements qui peuvent véritablement les rendre auteurs de nouvelles dynamiques institutionnelles (J. Ardoino, 1993).

\subsubsection{Fonction de régulation des décalages socioculturels}

Enfin, ouvrir l'analyse sur la régulation des décalages socioculturels conduit à travailler l'évolution de l'identité professionnelle de l'enseignant dans la société française, la question centrale étant celle de la rupture entre la constitution historique de cette identité, issue des idéaux de la Troisième République, et la réalité actuelle des attentes sociales de la jeunesse et des familles. Dans la tradition culturelle du rôle de l'école dans la société, bon nombre d'enseignants, pensent que l'éducation est essentiellement du ressort des familles, et que leur rôle consiste à enseigner à des élèves a priori déjà éduqués. Cette conception les conduit à verrouiller la dimension éducative de leur métier, ce qui provoque parfois des situations de crise tout à fait épuisantes.

87 L'enjeu est ici de travailler sur la nouvelle fonction sociale de l'École dans l'éducation du citoyen, en repositionnant sa mission éducative au regard de sa mission d'instruction. Pratiquement, cela oblige à reconsidérer fondamentalement la situation isolée de l'enseignant dans sa classe, pour ouvrir sur un travail d'équipe éducative, mettant en œuvre de manière coordonnée la construction dans l'établissement des repères sociaux fondamentaux.

\section{Conclusion}

L'ensemble du travail que nous venons de présenter s'inscrit dans la volonté d'intégrer les entrées théoriques et pratiques de la formation, en développant des dispositifs d'analyse de pratiques qui permettent d'étayer le transfert professionnel. Cette perspective assigne ces dispositifs à une double fonctionnalité :

- d'une part ils doivent assurer l'émergence des logiques intrinsèques de l'action située ;

- et d'autre part ils doivent permettre de modéliser ces logiques au regard de la culture professionnelle extrinsèque à cette action.

Cette double fonctionnalité ouvre sur deux champs théoriques de référence : celui de l'analyse de l'action située, et celui de la modélisation de cette action dans la culture professionnelle. Cette dualité théorique est parfois considérée comme paradoxale, en ce sens que l'on oppose de manière radicale les approches intrinsèque et extrinsèque des phénomènes d'enseignement. Dans une perspective anthropologique, et plus particulièrement en référence à l'analyse praxéologique avancée par Y. Chevallard (1997), nous rejetons cette radicalité épistémologique en nous plaçant dans une posture dialogique (E. Morin, 1990) que nous pensons indispensable à la formation professionnelle des enseignants. 


\section{BIBLIOGRAPHIE}

ALTET M. : «L'analyse de pratiques : une démarche de formation professionnalisante ? », in Recherche et formation, $\mathrm{N}^{\circ} 35,2000$, pp. 25-41.

ARDOINO J. : « L'approche multiréférentielle en formation et en sciences de l'éducation », in Pratiques de formation analyses, $\mathrm{N}^{\circ}$ 25-26. Paris, Service de formation permanente, Université Paris VIII, 1993.

BLANCHARD-LAVILLE Cl. et Coll. : Variations sur une leçon de mathématiques. Paris, Editions l'Harmattan, 1997.

BROUSSEAU G. : « Fondements et méthodes de la didactique des mathématiques », in Recherches en Didactique des mathématiques, Vol. 7, № 2, 1986, pp. 33-115.

BROUSSEAU G. : « Le contrat didactique : le milieu », in Recherches en Didactique des mathématiques, Vol. $9 \mathrm{~N}^{\circ} 3,1989$, pp. 309-326.

CHEVALLARD Y. : «Concepts fondamentaux de la didactique : perspectives apportées par une approche anthropologique ", in Recherches en Didactique des mathématiques, vol. $12 \mathrm{~N}^{\circ} 1,1992$, pp. 73-112.

CHEVALLARD Y.: « Analyse des pratiques enseignantes et didactique des mathématiques: l'approche anthropologique ", in Actes de l'université d'été, Analyse despratiques enseignantes et didactique des mathématiques (La Rochelle). Clermont Ferrand, Edités par l'IREM de Clermont Ferrand, juillet 1997.

CIFALI M. : « Des pratiques en récits », in Educateur, N² 1995.

CIFALI M. : « Démarche clinique, formation et écriture », in Paquay L., Altet M., Charlier E. et Perrenoud Ph. (dir), Former des enseignants professionnels. Quelles stratégies ? Quelles compétences? . Bruxelles (Belgique), De Bœck, 1996, pp. 119-135. COULON A. : Ethnométhodologie et éducation. Paris, PUF, 1993.

DEFRANCE B. : «L'école un lieu de non droit », in Actes du Forum Enseignement, Ecole de la démocratie, démocratie dans l'école, Bruxelles, Conseil de la communauté française, 1995. (Paru également dans le Journal des droits des jeunes, $\mathrm{N}^{\circ} 147$, septembre 1995, Paris.)

DOUADY R. : « Des apports de la didactique des mathématiques à l'enseignement ", in Repères, IREM, N 6, 1992, pp. 132-158.

ETIENNE R. et LEROUGE A. : Enseigner en collège ou en lycée repères pour un nouveau métier. Paris, Armand Colin (collection : formation des enseignants), 1997.

IMBERT F. : Médiatiations institutions et loi dans la classe. Paris, ESF, 1994.

LEROUGE A. : « Le conflit adulte enfant en milieu scolaire : éléments sur la formation des enseignants ", in Neuropsychiatrie de l'enfance et de l'adolescence, $46^{\mathrm{e}}$ année, $\mathrm{N}^{\circ} 7-8,1998$, pp. 373-378.

LERBET-SERENI F. et VIOLET D. : « Paradoxes de la médiation. Tradition et alternance », in Revue Française de Pédagogie, $\mathrm{N}^{\circ} 128,1999$, pp. 17-24. 
MORIN E. : Introduction à la pensée complexe. Paris, ESF (Collection : Communication et complexité), 1990.

PERRENOUD Ph. : « De la pratique réflexive au travail sur l'habitus », in Recherche et formation, N $\circ 36,2001$.

POSTIC M. : Observation et formation des enseignants. Paris, PUF, 1977.

THEUREAU J. : Le cours d'action : analyse sémio-logique. Essai d'une anthropologie cognitive située . Berne (Suisse), Peter Lang, 1992.

VERGNAUD G. : « La théorie des champs conceptuels », in Recherches en Didactique des mathématiques, Vol. $10 \mathrm{~N}^{\circ} 2-3,1991$, pp. 135-169.

VERGNAUD G. et LABORDE C. : «L'apprentissage et l'enseignement des mathématiques », in Apprentissages et Didactiques, où en est-on ? Paris, Hachette Education, 1994.

VYGOTSKI L.S. : Pensée et Langage. Messidor Editions sociales, 1985 (1re édition en 1934).

\section{NOTES}

1. Pour assurer une dévolution d'usage de cette notion d'habitus (Ph. Perrenoud, 2001), nous la traduisons par l'expression « réflexe professionnel » qui marque bien le caractère automatique et pré conscient des schèmes activés en situation.

2. Il paraît important de préciser qu'il ne s'agit pas d'une vision dichotomique du sujet et de la culture, mais d'une modélisation de l'état entre le culturel et le personnel à un moment de l'histoire du sujet. Dans cette perspective, le versant sujet peut être appréhendé comme l'ensemble du culturel professionnel qu'il a déjà intégré au moment de la formation.

3. Macro praxéologie didactique visant à faire construire une nouvelle notion à partir des représentations déjà installées chez les élèves.

4. Macro praxéologie pédagogique visant à faire s'approprier à la classe les règles de son fonctionnement interne.

5. Ce type de travail ne peut se faire au cours de l'entretien, mais on peut très bien l'envisager dans un entretien complémentaire, ou comme exercice de formation dans le cadre d'un mémoire professionnel.

6. Le lecteur intéressé et non spécialiste de didactique des mathématiques pourra se référer au chapitre "L'apprentissage et l'enseignement des mathématiques", in Apprentissages et Didactiques, où en est-on ? Hachette Education, 1994.

7. Cf., L'ouvrage d'ETIENNE $R$ et LEROUGE A : Enseigner en collège ou en Lycée, repères pour un nouveau métier. Paris, Armand Colin, 1997.

\section{RÉSUMÉS}

Cet article présente un dispositif d'analyse de pratiques développé à l'IUFM de Montpellier, dans l'objectif d'intégrer les aspects théoriques de la formation à la pratique professionnelle observée sur le terrain. La démarche se fonde sur la notion de praxéologie, autour de laquelle est construit le protocole expérimental. Ce protocole consiste à dégager de l'observation d'un enseignant en 
situation des séquences fonctionnelles de gestes professionnels, puis à développer sur ces séquences un repérage théorique extrinsèque multiréférencé.

This article presents a course of action for the analysis of professional practices, which has been developed at the Montpellier IUFM (teacher training college), with the goal of integrating the theoretical aspects of teacher training with professional practice as observed in the field. This approach is grounded upon the notion of praxéology on the basis of which this experimental protocol is constructed. This protocol consists in drawing out from the observation of teacher «in situ» functional sequences of professional acts, then, in building upon these sequences a multireferential and extrinsic theoretical framework.

INDEX

Keywords : analysis of teaching practices, in situ actions, pedagogical advice, praxéology, teacher training

Mots-clés : action située, analyse de pratiques, conseil pédagogique, formation des maîtres, praxéologie

\section{AUTEUR}

\section{ALAIN LEROUGE}

Equipe LIRDEF, IUFM de Montpellier 